\title{
Health care and social media: What patients really
}

\section{understand [version 1; peer review: 2 approved]}

\section{Kyle Hoedebecke (D1), Lindsey Beaman², Joy Mugambi (iD)3, Sanam Shah4, Marwa Mohasseb ${ }^{5}$, Cheyanne Vetter ${ }^{6}$, Kim Yu ${ }^{7}$, Irini Gergianaki ${ }^{8}$, Emily Couvillon ${ }^{9}$}

${ }^{1}$ WONCA Polaris, Seoul, South Korea

2Leidos Biomedical Research, Inc., Frederick, MD, USA

${ }^{3}$ Afriwon Renaissance Movement, Naivasha, Kenya

${ }^{4}$ The Spice Route Movement, Karachi, Pakistan

${ }^{5} \mathrm{Al}$ Razi Movement, Cairo, Egypt

${ }^{6}$ WONCA Polaris, Saskatchewan, Canada

${ }^{7}$ Covenant Healthcare, Frankenmuth, MI, USA

${ }^{8}$ University of Crete, Crete, Greece

${ }^{9}$ Texas Medical Center Library, Houston, TX, USA

V1 First published: $08 \mathrm{Feb} 2017,6: 118$

https://doi.org/10.12688/f1000research.10637.1

Latest published: 08 Feb 2017, 6:118

https://doi.org/10.12688/f1000research.10637.1

\section{Abstract}

Background: Low health literacy is associated with decreased patient compliance and worse outcomes - with clinicians increasingly relying on printed materials to lower such risks. Yet, many of these documents exceed recommended comprehension levels. Furthermore, patients look increasingly to social media (SoMe) to answer healthcare questions. The character limits built into Twitter encourage users to publish small quantities of text, which are more accessible to patients with low health literacy. The present authors hypothesize that SoMe posts are written at lower grade levels than traditional medical sources, improving patient health literacy.

Methods: The data sample consisted of the first 100 original tweets from three trending medical hashtags, leading to a total of 300 tweets. The Flesch-Kincaid Readability Formula (FKRF) was used to derive grade level of the tweets. Data was analyzed via descriptive and inferential statistics. Results: The readability scores for the data sample had a mean grade level of 9.45 . A notable $47.6 \%$ of tweets were above ninth grade reading level. An independent-sample t-test comparing FKRF mean scores of different hashtags found differences between the means of the following: \#hearthealth versus \#diabetes $(t=3.15, p$ $=0.002)$; \#hearthealth versus \#migraine $(\mathrm{t}=0.09, \mathrm{p}=0.9)$; and \#diabetes versus \#migraine $(t=3.4, p=0.001)$. Conclusions: Tweets from this data sample were written at a mean grade level of 9.45 , signifying a level between the ninth and tenth grades. This is higher than desired, yet still better than traditional sources, which have been previously analyzed. Ultimately, those responsible for health care SoMe posts must continue to improve efforts to reach the

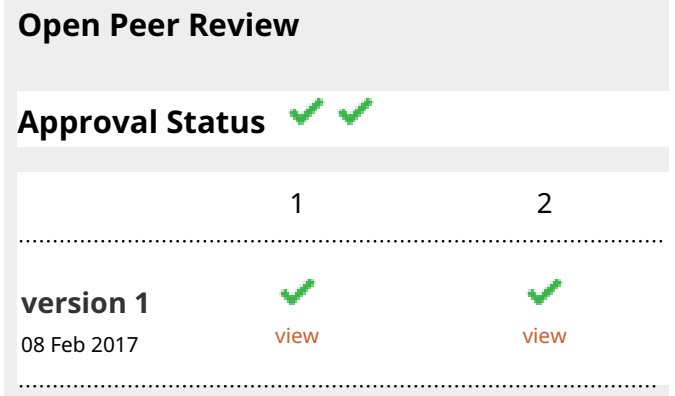

1. Shailendra Prasad, University of Minnesota, Minneapolis, USA

2. Shabir A. H. Moosa (D), University of the Witwatersrand, Johannesburg, Johannesburg, South Africa

Any reports and responses or comments on the article can be found at the end of the article. 
recommended reading level (between the sixth and eighth grade), so as to ensure optimal comprehension of patients.

\section{Keywords}

Social Media, Twitter, Web 2.0, health literacy, patient comprehension

Corresponding author: Kyle Hoedebecke (khoedebecke@gmail.com)

Competing interests: No competing interests were disclosed.

Grant information: The author(s) declared that no grants were involved in supporting this work.

Copyright: @ 2017 Hoedebecke $\mathrm{K}$ et al. This is an open access article distributed under the terms of the Creative Commons Attribution License, which permits unrestricted use, distribution, and reproduction in any medium, provided the original work is properly cited. Data associated with the article are available under the terms of the Creative Commons Zero "No rights reserved" data waiver (CC0 1.0 Public domain dedication).

How to cite this article: Hoedebecke K, Beaman L, Mugambi J et al. Health care and social media: What patients really understand [version 1; peer review: 2 approved] F1000Research 2017, 6:118 https://doi.org/10.12688/f1000research.10637.1

First published: 08 Feb 2017, 6:118 https://doi.org/10.12688/f1000research.10637.1 


\section{Introduction}

Health literacy - defined as the degree to which an individual has the capacity to obtain, communicate, process, and understand basic health information and services to make appropriate health decisions - is considered to be the single best predictor of an individual's health status (http://www.cdc.gov/healthliteracy/ learn/ $)^{1}$. Low health literacy correlates with decreased patient compliance and poorer outcomes, leading to an increase in clinician reliance on printed materials to mitigate such risks ${ }^{2}$. Yet, a recent study identified that many of these materials exceed the recommended sixth to eighth grade reading level of the American Medical Association (AMA), National Institute of Health (NIH) and Center for Disease Control and Prevention (CDC) (http://www. nlm.nih.gov/medlineplus/etr.html; http://www.cdc.gov/DHDSP/ cdcynergy_training/Content/activeinformation/resources/simpput. pdf $)^{3,4}$. As medical vocabulary becomes more integrated into social media (SoMe), the healthcare community must remember to employ comprehensible language when engaging audiences through platforms such as Facebook, Twitter, and LinkedIn.

Generally, patients are increasingly relying on SoMe as a primary avenue for answering healthcare questions ${ }^{5,6}$. For example, this may be due to the character limits built into Twitter that encourage users to publish small chunks of text, which are increasingly accessible to patients with low health literacy ${ }^{7}$. As health literacy directly impacts patient outcomes, it remains imperative for healthcare providers to intentionally tailor their writing level of SoMe posts to enhance patient-centred communication and comprehension.

The present authors hypothesized that SoMe posts on the Twitter platform are written at a lower grade level than traditional medical sources, allowing for better patient health literacy.

\section{Methods}

The data sample consisted of the first 100 original tweets in 2016 via the pay-to-access Symplur Signal analytics tools (http://www. symplur.com/signals/) from each of the March 2016 top trending hashtags: \#hearthealth, \#diabetes and \#migraines, leading to a total of 300 tweets being analyzed. Trending hashtags related to primary care were selected, as these tweets would have the greatest impact and overall reach worldwide. Exclusion criteria included nonEnglish or non-medical tweets, as well as those that encompassed links with non-medical webpages or product advertisements.

The Flesch-Kincaid Readability Formula (FKRF) is a validated tool to assess the grade level of written material and is calculated with the following formula: $206.835-1.015$ (total words/total sentences) - 84.6 (total syllables/total words). The FKRF Grade Level Scores can be interpreted as shown in Table 1 between the fifth grade to graduate levels ${ }^{8}$. Each tweet was evaluated via FKRF to derive grade level. SPSS (version 21.0 for Mac; http://www.ibm. com/analytics/us/en/technology/spss/) was used for data analysis, and data was analyzed using descriptive and inferential statistics. Descriptive statistics included the mean with $95 \%$ confidence interval, median, range and standard deviation of FKRF scores. All $p$ values were derived from two-sided $t$-tests. The project was approved by Stanford's IRB and Medical Ethics Team, as a part of the 2016 Stanford MedX/Symplur Social Media Competition.

\section{Results}

The readability scores for the 300 total tweets evaluated are presented in Table 2. The mean FKRF grade level was 9.45, signifying a level between the ninth and tenth grades. A notable $47.6 \%$ of tweets were above the ninth grade reading level (Table 2). There was a wide range of FKRF scores, as shown in Table 3, varying from elementary to postgraduate levels.

\begin{tabular}{|c|c|}
\hline $\begin{array}{l}\text { Flesch-Kincaid } \\
\text { grade level score }\end{array}$ & $\begin{array}{l}\text { Reading difficulty } \\
\text { rating }\end{array}$ \\
\hline 5 th & Very easy to read \\
\hline 6 th & Easy to read \\
\hline 7th & Fairly easy to read \\
\hline 8th and 9th & $\begin{array}{l}\text { Plain English/ } \\
\text { standard }\end{array}$ \\
\hline 10th to 12 th & $\begin{array}{l}\text { Fairly difficult to } \\
\text { read }\end{array}$ \\
\hline College/13th-16th & Difficult to read \\
\hline $\begin{array}{l}\text { College graduate } \\
\text { and beyond }\end{array}$ & $\begin{array}{l}\text { Very difficult to } \\
\text { read }\end{array}$ \\
\hline
\end{tabular}

Table 2. Flesch-Kincaid Readability Formula (FKRF) grade level scores for the total sample.

\begin{tabular}{|l|l|}
\hline $\begin{array}{l}\text { Total sample } \\
(\mathbf{n}=\mathbf{3 0 0})\end{array}$ & $\begin{array}{l}\text { FKRF grade } \\
\text { level }\end{array}$ \\
\hline Mean & 9.45 \\
\hline Median & 9.05 \\
\hline $\begin{array}{l}\text { Standard } \\
\text { deviation }\end{array}$ & 4.95 \\
\hline Range & $1.2-28.4$ \\
\hline
\end{tabular}

Table 3. Tweet material grade level summary by Flesch-Kincaid Readability Formula (FKRF) $(n=300)$.

\begin{tabular}{|l|l|}
\hline Grade level & FKRF, $\mathbf{n}(\%)$ \\
\hline 1st -3 rd & $32(10.7)$ \\
\hline 4th -6 th & $59(19.7)$ \\
\hline 7th -9 th & $66(22.0)$ \\
\hline 10th - 12th & $70(23.3)$ \\
\hline$>12$ th & $73(24.3)$ \\
\hline
\end{tabular}


An independent-sample t-test comparing the FKRF mean scores of different hashtags found differences between the means of groups as follows: \#hearthealth versus \#diabetes $(t=3.15$, $p=0.002)$; \#hearthealth versus \#migraine $(t=0.09, p=0.9)$; and \#diabetes versus \#migraine $(t=3.4, p=0.001)$. Therefore, there was a significant difference between the means of two groups: \#hearthealth versus \#diabetes, and \#diabetes versus \#migraine. Although it is unclear why the differences exist, this identifies that the grade level comprehension varies significantly when dealing with tweets surrounding differing health issues. One such explanation could be the differing characteristics of the tweet author and their health care experience. Additionally, the differing incidences of migraines and heart disease may affect the availability of reading materials as well as the grade level at which each is written.

Dataset 1 . The 300 tweets analysed by the present study divided by \#migraine, \#hearthealth and \#diabetes

http://dx.doi.org/10.5256/f1000research.10637.d150437

Dataset 2. Raw data for SPSS

http://dx.doi.org/10.5256/f1000research.10637.d150438

\section{Discussion}

SoMe - especially Twitter - is a cost-effective, interactive communication tool with increasing applicability within the medical sector ${ }^{9}$. Although limited health literacy of the audience poses a real threat in disseminating health messages, few studies have examined the readability of Twitter healthcare posts for the general public. In the present study, the authors found that a Twitter sample $(n=300)$ was written at a mean of FKRF grade level 9.45, signifying a level between the ninth and tenth grade (Table 1). This outcome proves much closer to the NIH readability goal, as compared to previous studies that found patient medical consent forms to be written between the eleventh to thirteenth grade levels (three to five grades higher than the current NIH recommendation), and on major associations' websites and educational materials, which were written above the recommended reading level (http://www.nlm.nih.gov/medlineplus/etr.html).

One potential reason for this outcome lies in Twitter's character limit itself, which permits only 140 characters to be written. Undoubtedly, this may prove a double-edged sword as this limitation creates a more manageable length, but also forces the composer to employ more concise terminology carrying a more complex readability factor. Given the increasing number of Twitter users, readability should be further evaluated towards meaningful health messaging, diminishing disparities in comprehension and ameliorating patient difficulties to understand and follow instructions and recommendations.

This study has some limitations, including the relatively small sample, the use of a single readability scale and a single SoMe platform. On the other hand, there are major strengths, as our study provides an updated focus of readability of web 2.0 communication tools. The findings highlight the possibility that Twitter can be a way of reaching the readability guidelines, as compared to written educational materials or online materials on websites. Twitter was used as a model, but more platforms on SoMe should be evaluated, so that guidelines could be shaped to recognize the unmet needs of health communication in a modern era. Ultimately, those responsible for health care SoMe and other relevant platforms posts must continue to improve efforts to reach the recommended reading level, so as to ensure optimal comprehension and enhance the capacity of patients and doctors to mutually interact.

\section{Conclusions}

The sample studied identifies that health care SoMe posts allow for better patient health literacy than traditional medical sources. Health care advocates must remain vigilant, so that posts improve upon current readability levels. Lastly, respectable medical sources should consider additional use of SoMe avenues to dispense more comprehensible health care information to a wider patient audience.

\section{Data availability}

Dataset 1: The 300 tweets analysed by the present study divided by \#migraine, \#hearthealth and \#diabetes. doi, 10.5256/ f1000research.10637.d150437 10

Dataset 2: Raw data for SPSS. doi, 10.5256/f1000research.10637. d150438 ${ }^{11}$

\section{Author contributions}

$\mathrm{KH}$ - lead author, team leader, wrote background; LB - data collection and grade level interpretation, wrote the results section; SS- statistical analysis and wrote methods/results section; KMdata collection, wrote the results section; EC - literature search, background writer; MM - statistical analysis and wrote methods/ results section; JM - data collection, wrote the results section; CV - data collection, wrote the results section; IG - statistical analysis, wrote the results/conclusion section. All authors edited and approved the final content of the manuscript.

\section{Competing interests}

No competing interests were disclosed.

\section{Grant information}

The author(s) declared that no grants were involved in supporting this work.

\section{Acknowledgements}

A presentation of the results of this study was a global semifinalist for the 2016 Stanford-MedX/Symplur Healthcare Social Media Competition. Accepted for presentation at the March 2017 Uniformed Service Academy of Family Physicians Conference (Seattle, USA). 
1. Baker DW, Parker RM, Williams MV, et al.: The relationship of patient reading ability to self-reported health and use of health services. Am J Public Health. 1997; 87(6): 1027-30.

PubMed Abstract | Publisher Full Text | Free Full Text

2. Berkman ND, Sheridan SL, Donahue KE, et al:: Health literacy interventions and outcomes: An updated systematic review. Evid Rep Technol Assess (Full Rep). 2011; (199): 1-941.

PubMed Abstract | Free Full Text

3. Berry-Caban CS, Portee CL, Beaman LA, et al:: Readability of research consent forms in a military treatment facility. Ethics \& Medicine: An International Journal of Bioethics. 2014; 30(2): 109-117.

Reference Source

4. Weiss B: Health Literacy: A Manual for Clinicians. Chicago, IL: American Medical Association, American Medical Foundation, 2003. Reference Source

5. Fox S: The social life of health information, 2011. Pew Research Center: Internet, Science \& Tech, 2011

Reference Source
6. McInnes N, Haglund BJ: Readability of online health information: Implications for health literacy. Inform Health Soc Care. 2011; 36(4): 173-189.

PubMed Abstract | Publisher Full Text

7. Bell J III, Mertz J: Hashtags and health literacy: How social media transforms engagement. HIMSS Patient Literacy and Health IT Work Group. 2015. Reference Source

8. Calderón JL, Morales LS, Liu H, et al:: Variation in the readability of items within surveys. Am J Med Qual. 2006; 21(1): 49-56.

PubMed Abstract | Publisher Full Text | Free Full Text

9. Lee JY, Sundar SS: To tweet or to retweet? That is the question for health professionals on twitter. Health Commun. 2013; 28(5): 509-524.

PubMed Abstract | Publisher Full Text

10. Hoedebecke K, Beaman L, Mugambi J, et al.: Dataset 1 In: Health care and social media: What patients really understand. F1000Research. 2017. Data Source

11. Hoedebecke K, Beaman L, Mugambi J, et al:: Dataset 2 In: Health care and social media: What patients really understand. F1000Research. 2017. Data Source 


\section{Open Peer Review}

\section{Current Peer Review Status:}

\section{Version 1}

Reviewer Report 30 March 2017

https://doi.org/10.5256/f1000research.11463.r20791

(C) 2017 Moosa S. This is an open access peer review report distributed under the terms of the Creative Commons Attribution License, which permits unrestricted use, distribution, and reproduction in any medium, provided the original work is properly cited.

\section{Shabir A. H. Moosa}

Department of Family Medicine, University of the Witwatersrand, Johannesburg, Johannesburg, South Africa

Congrats to this global set of young authors from the Wonca circle! Really good application of research method to an important issues.

There are some suggestions that would help the authors.

1. The authors say " present authors hypothesize that SoMe posts are written at lower grade levels than traditional medical sources, improving patient health literacy". The research method, results, and discussion do not reflect that with no clarity as to what "traditional medical sources" are compared with. Reference to previous studies under discussion is referenced to the MedlinePlus webpage on "How to write..." with no clear evidence of 'traditional medical sources' readability scores with the FKRF. Printed material and websites would have been a useful comparator vs. consent forms. The comparison of different hashtags and statistical tests seem irrelevant/tangential to the hypothesis or enquiry.

2. The choice of Twitter and assumptions about it ie. small chunks of text making it more accessible are contradicted later when the Twitter limitations of complex readability factor are later related due to small chunks. It would have been useful to relate to global use of SoMe platforms, by population and possibly patients.

3. The method of using the FKRF is not clearly articulated and seems difficult to relate to Table 2 readability score.

4. The conclusion "The sample studied identifies that health care SoMe posts allow for better patient health literacy than traditional medical sources." seems largely unsupported.

Keep it up. 
Competing Interests: No competing interests were disclosed.

I confirm that I have read this submission and believe that I have an appropriate level of expertise to confirm that it is of an acceptable scientific standard.

Reviewer Report 07 March 2017

https://doi.org/10.5256/f1000research.11463.r20061

(C) 2017 Prasad S. This is an open access peer review report distributed under the terms of the Creative Commons Attribution License, which permits unrestricted use, distribution, and reproduction in any medium, provided the original work is properly cited.

\section{Shailendra Prasad}

Dept of Family Medicine and Community Health, University of Minnesota, Minneapolis, MN, USA

I compliment the authors on this study. It is fascinating to see the group from various parts of the world collaborate on this project.

- The project/manuscript is a much-needed one with the rapid growth of social media in society. There are indications that access to social media platforms have increased dramatically in the last decade and further democratizes information sharing.

- The authors use a novel approach to look at the content of various social media posts in Twitter regarding health and determine that the readability of the posts are much better than conventional health education methods.

- The article is well written and easily readable.

I have a couple of minor corrections to recommend.

1) Highlight that this analysis is particular to Twitter. The authors have done this, but I would recommend reinforcing this as the title indicates Social Media ( which has different platforms).

2) The issue then is possibly of brevity rather than the platform. I would recommend that the authors highlight this in the discussion too.

3) I recommend that the authors highlight the other issues in understanding healthrelated information, including the assimilation of the information and behavior change that needs to follow and that further studies should look at this.

Again, my compliments to the authors.

Competing Interests: No competing interests were disclosed.

I confirm that I have read this submission and believe that I have an appropriate level of expertise to confirm that it is of an acceptable scientific standard. 
The benefits of publishing with F1000Research:

- Your article is published within days, with no editorial bias

- You can publish traditional articles, null/negative results, case reports, data notes and more

- The peer review process is transparent and collaborative

- Your article is indexed in PubMed after passing peer review

- Dedicated customer support at every stage

For pre-submission enquiries, contact research@f1000.com 